\title{
A Disciplined Approach to Aspect Composition
}

\author{
Roberto Lopez-Herrejon \\ Computing Laboratory \\ Oxford University \\ Oxford, England, OX1 3QD \\ rlopez@comlab.ox.ac.uk
}

\author{
Don Batory \\ Department of Computer Sciences \\ University of Texas at Austin \\ Austin, Texas, 78712 U.S.A. \\ batory@cs.utexas.edu
}

\author{
Christian Lengauer \\ Fakultät für Mathematik und Informatik \\ Universität Passau \\ Passau, Germany \\ lengauer@fmi.uni-passau.de
}

\begin{abstract}
Aspect-oriented programming is a promising paradigm that challenges traditional notions of program modularity. Despite its increasing acceptance, aspects have been documented to suffer limited reuse, hard to predict behavior, and difficult modular reasoning. We develop an algebraic model that relates aspects to program transformations and uncovers aspect composition as a significant source of the problems mentioned. We propose an alternative model of composition that eliminates these problems, preserves the power of aspects, and lays an algebraic foundation on which to build and understand AOP tools.
\end{abstract}

\section{Introduction}

Aspect-oriented programming $(A O P)$ is a promising paradigm that challenges and enhances traditional notions of program modularity [20]. It has been widely applied to different languages but the most influential implementation is AspectJ [8][20][29]. AspectJ has sophisticated and powerful modularization mechanisms that bring clear benefits over traditional modules but also has equally significant drawbacks. Aspects have been documented to suffer limited reuse [22], hard to predict behavior [35], and difficult modular reasoning [16][1]. All these factors hinder useful software engineering practices such as step-wise development [46] and its natural materialization in component-based software engineering (CBSE) [44], where programs are developed incrementally by composing components one at a time.

An aspect is a declaration of changes that are to be made to a program; the process of making these changes is called weaving. There have been several proposals to define aspect semantics [19]. The most common uses an event-based model [45]. There are many ways in which aspects and weaving can be implemented. The historical roots of AOP are in

Permission to make digital or hard copies of all or part of this work for personal or classroom use is granted without fee provided that copies are not made or distributed for profit or commercial advantage and that copies bear this notice and the full citation on the first page. To copy otherwise, or republish, to post on servers or to redistribute to lists, requires prior specific permission and/or a fee.

PEPM '06, January 9-10, 2006, Charleston, South Carolina, USA.

Copyright 2006 ACM 1-59593-196-1/06/0001 ...\$5.00. meta-object protocols (MOPs) [14]. A MOP transforms the metaclasses that control the behavior of a program's execution. On the other hand, aspect compilers perform static weaving by producing woven binaries or woven source [5]. When a woven binary is run, its execution flow is indistinguishable from that of a MOP implementation. Aspect compilers are popular today because, among other reasons, they offer improved program run-time performance through static optimizations that are too expensive to realize via MOPs [6].

If we want to understand the impact that aspects have on a program's structure, we need to study an implementation of aspects that makes program structure explicit. This is where transformations come in. A program transformation is a function that maps programs to programs [36]. While there are few aspect compilers [21] that explicitly use program transformation tools [41], we claim that the effects of static weaving can be understood in terms of transformations. This connection enables us to raise aspects from code artifacts to mathematical entities (functions from programs to programs) and develop algebraic models of aspects and their composition. These models reveal aspect composition as a significant source of the problems mentioned above. We have demonstrated that functional composition is essential to the synthesis of large-scale programs [10][11]. To the best of our knowledge, large-scale program synthesis has not been tackled yet with AOP technology [3]. We propose an alternative model of aspect composition, based on function composition, that eliminates the above problems while preserving the power of AspectJ. We believe our model lays an algebraic foundation on which to build and understand AOP tools.

\section{AspectJ Overview}

AspectJ [7] is an extension of Java whose goal is to modularize aspects, concerns that crosscut traditional module boundaries such as classes and interfaces, that would otherwise be scattered and tangled with the implementation of other concerns [8]. AspectJ has two types of crosscuts, static and dynamic, that we illustrate and interpret as transformations.

\subsection{Static Crosscuts}

Static crosscuts affect the static structure of a program [8][29]. We focus on introductions, also known as inter-type declarations, that add fields, methods, and constructors to 
existing classes and interfaces. In AspectJ, standard Java classes and interfaces are referred to as base code. Consider class point defined below:

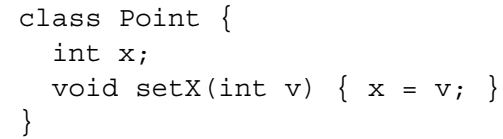

The following aspect TwoD adds (introduces) a second coordinate value to class Point. It adds field $y$ and method set $\mathrm{Y}$ :

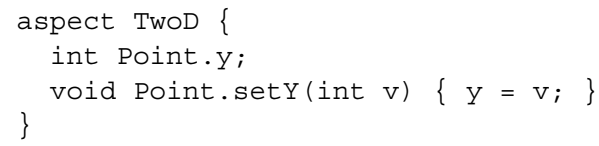

When these two files are composed or woven by the AspectJ compiler ajc using the command:

ajc Point.java TwoD.java

The result is a new class Point' with the introduced members underlined below:

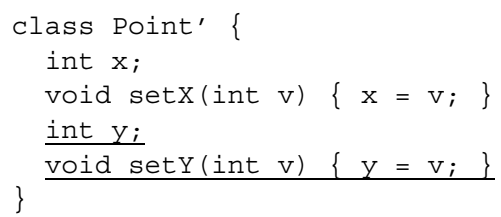

AspectJ generally uses more sophisticated rewrites than those shown in this paper. The composed code snippets we present simplify illustration and are behaviorally equivalent to those produced by ajc.

Static Crosscuts as Transformations. From the program transformation perspective, base code such as Point in (1) represents a value to which a function (a program transformation) or aspect is applied. For instance, class Point' in (2) can be written as the following expression:

Point' = TwoD ( Point)

That is, Point is a base program and TwoD is a function that maps Point to Point'.

\subsection{Dynamic Crosscuts}

Dynamic crosscuts, in contrast, run additional code when certain events occur during program execution. The semantics of dynamic crosscuts are commonly understood and defined in terms of an event-based model [30][45]. As a program executes, different events fire. These events are called join points. Examples of join points are: variable reference, variable assignment, execution of a method body, method call, etc. A pointcut is a predicate that selects a set of join points. Advice is code executed before, after, or around each join point matched by a pointcut.

The following aspect is the familiar logging example. Its interpretation is: run the advice code (underlined) after (advice type) the execution of methods in class Point whose name starts with 'set' (pointcut in italics).

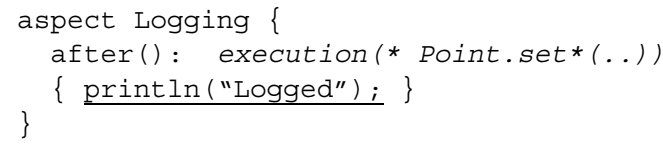

From a compiler perspective, an equivalent interpretation is: insert the advice code after the body of any method in class Point whose name starts with 'set'. For example, if aspect Logging is woven into class Point' in (2) the result is equivalent to:

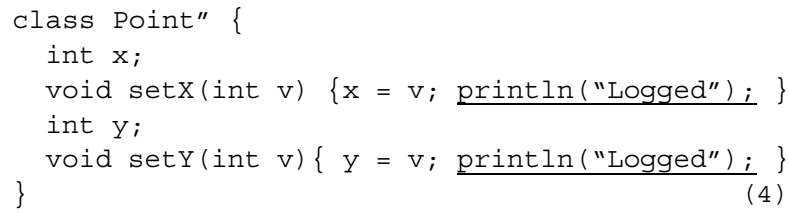

Dynamic Crosscuts as Transformations. Dynamic crosscuts can be implemented by transformations. For example, class Point" in (4) can be written as the expression:

Point" = Logging (Point') = Logging(TwoD(Point))

That is, class Point" is the result of applying two transformations, or from an AOP perspective the result of weaving two aspects, into class Point.

AspectJ provides an array of sophisticated mechanisms to define powerful pointcuts and to perform complex rewrites when weaving aspects into programs. All dynamic crosscuts can be understood as transformations including pointcut designators such as cflow, args, this, and target, which expose context information of a join point [8].

Consider cflow(Y) where $\mathrm{Y}$ is a pointcut. Suppose y captures a specific method execution or method call. cflow $(Y)$ is the set of join points that occur during the execution of $Y$, from the time that the method is called to the time of the return [8]. An interesting question to ask is if a join point $x$ occurs within the control flow of $\mathrm{Y}$ ? The pointcut that expresses this is concisely written in AspectJ as:

Cflow ( Y ) \&\& pointcut_for_X

From a compiler's perspective, control flow advice is a transformation that is composed from four simple transformations: (i) introduce a control flow stack $s$, (ii) before each $\mathrm{Y}$ join point, push a marker $\mathrm{m}$ on $\mathrm{S}$, and (iii) after each $\mathrm{Y}$ join point, pop $\mathrm{m}$ off $\mathrm{S}$. For the duration that $\mathrm{m}$ is on $\mathrm{S}$, any join point that occurs does so within the control flow of $y$. And finally, (iv) at each $\mathrm{x}$ join point, check to see if $\mathrm{m}$ is on $\mathrm{s}$; if so, execute the advice code.

Aspect compilers, such as ajc, demonstrate that aspects can be implemented by transformations: ajc takes a base program and aspects as input and produces a woven binary as output. Even so, the connection of dynamic crosscuts, espe- 
cially cflow, to transformations remains controversial [23]. However, when given proper consideration, optimization and weaving techniques such as those presented in [6][24][34] are examples of program transformations, sophisticated indeed, but transformations nonetheless.

\subsection{Advice Precedence}

Recognizing that aspects can be realized as program transformations is a key first step in understanding how aspects impact program structure. The next step is to see how aspects are composed.

Multiple pieces of advice can be applied to the same join point. Advice precedence determines the order in which advice is woven. AspectJ deals with precedence differently depending on where the pieces of advice are defined, either in the same aspect or in different aspects [8].

Ordering aspects. AspectJ programmers have the option of declaring the order in which aspects are woven by a precedence statement such as:

declare precedence: Aspect 3, Aspect $_{2}$, Aspect $_{1}$;

In the above example, the advice of Aspect $_{1}$ is woven first, then the advice of Aspect $_{2}$, and finally the advice of Aspect ${ }_{3}$. If no precedence statement is declared, the precedence of aspects is undefined in the semantics of AspectJ. In such cases, the AspectJ compiler chooses an order in which to weave aspects. In general, this order cannot be inferred by programmers prior to weaving.

Unfortunately, different weaving orders can result in programs that behave differently. We need to know the weaving order to be able to predict the result. If the weaving order is undetermined, as in the absence of a precedence declaration, the woven program will at the least be not portable (since different compilers can choose different weaving orders). Moreover, programmers will not be informed about the order the compiler chooses, i.e., they will find it hard to predict the result of a weaving [4].

Ordering advice. Within an aspect, different pieces of advice appear in a certain textual order. However, the precedence of advice is governed by the following rules copied verbatim from [8]:

If two pieces of advice are defined in the same aspect, then there are two cases:

- If either are after advice, then the one that appears later in the aspect has precedence over the one that appears earlier.

1. The mathematical concept of precedence has the opposite meaning of precedence in AspectJ. Higher precedence in AspectJ means apply later, whereas mathematical precedence means apply earlier.
- Otherwise, then the one that appears earlier in the aspect has precedence over the one that appears later.

These precedence rules lead to two problems: 1) they may introduce a circularity such that the compiler cannot decide with which piece of advice to start the weaving, and 2) they cannot express all composition (weaving) orders.

The circularity problem is well-known [8], but the latter is not. A single aspect with three pieces of advice (identified by subscripts) illustrates both:

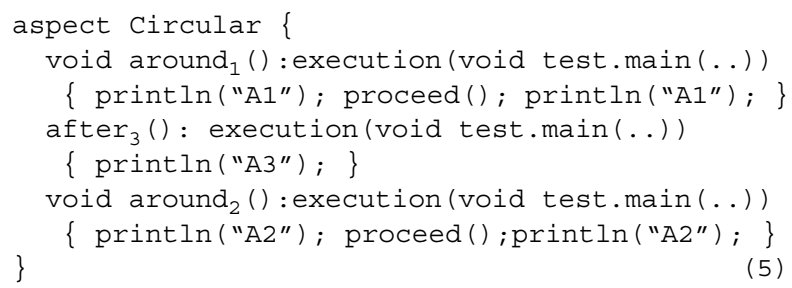

First, when the rules are applied, a circular precedence is created, as illustrated in the diagram to the right. To resolve the problem, programmers must manually modify

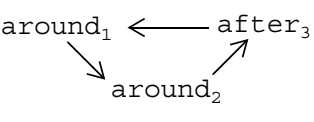
$\mathrm{X} \stackrel{\text { has precedence over }}{\longrightarrow} \mathrm{Y}$ the order in which the advice is listed in the program text, ensure that the resulting weaving order eliminates circularity, and produce a semantically appropriate weaving for the task at hand, a non-trivial and lengthy process.

Second, some composition orders cannot be attained. Suppose we want the following output sequence (A2, A1, <main>, A1, A3, A2), which is achieved by weaving around ${ }_{1}$ first, then after 3 , and then around 2 . In what order should advice around $_{1}$, after ${ }_{3}$, and around ${ }_{2}$ be listed in a single aspect file to achieve this weaving order?

The above rules dictate that around ${ }_{2}$ must be listed before around $_{1}$ (because around ${ }_{1}$ must be woven first). Advice after $_{3}$ must also appear before around $_{2}$ (to weave after 3 first). Thus, the ordering so far is: after ${ }_{3}$ then around $_{2}$ then around $_{1}$. But after must also appear after around $_{1}$ (for around $_{1}$ to be woven before after ${ }_{3}$ ). It is impossible for

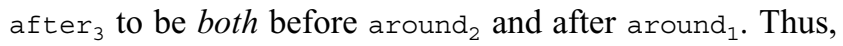
no linear ordering of the advice $\operatorname{around}_{1}$, around ${ }_{2}$, and after $_{3}$ can achieve the desired weaving order.

A way to realize such a weaving is to store each advice in a separate aspect file and use declare precedence:

declare precedence: around 2 , after 3 , around ${ }_{1}$;

Another way might be to convert all after and before advice into around advice, which can be easily ordered. But this then begs the question of why after and before advice have different ordering rules than around advice.

In summary, the current rules for precedence makes program reasoning unnecessarily difficult. But precedence is 
not the only problem with aspect composition. Fundamental software engineering practices such as step-wise development are not satisfactorily supported by AspectJ, as the following section shows.

\section{An Incremental Development Example}

Incremental or step-wise development (SWD) is a fundamental programming practice [11][44][46]. It aims at building complex programs from simpler ones by progressively adding programmatic details. SWD was not fully appreciated by the software engineering community for years. Today it is a centerpiece of core results in the synthesis of programs in product-lines [11] and component-based software engineering [44].

We illustrate a small but typical example of incremental development. We use subscripts to denote a particular version of our program at a given step and underline the code that is added by each increment.

Base. Class Point ${ }_{0}$ defines a 1 -dimensional point with an $\mathrm{x}$ coordinate and corresponding set $\mathrm{x}$ method:

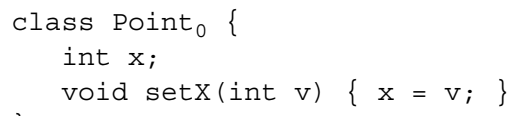

First increment. Adds coordinate $y$ and its sety method to Point $_{0}$. The result is:

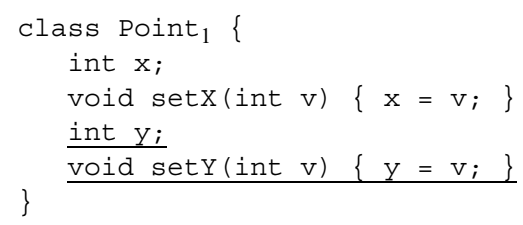

Second increment. Counts how many times the set methods are executed. Adding both increments to base yields:

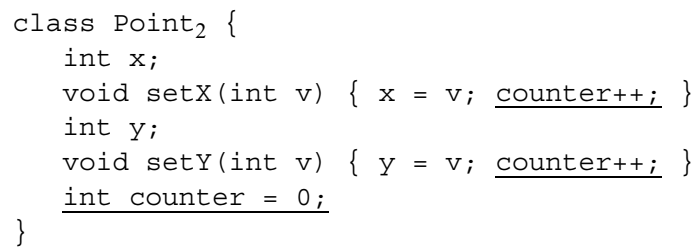

Third increment. Adds a color field and its corresponding set method to Point 2 :

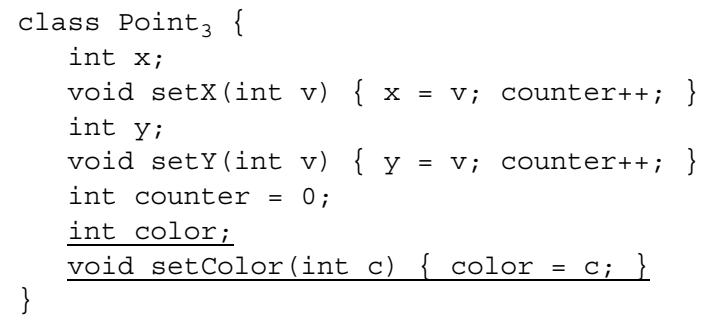

Now here is an implementation in AspectJ:
Base. Identical to (6) because classes are the base code of AspectJ applications.

First increment. We define aspect TwoD that introduces field $y$ and method sety to class Point:

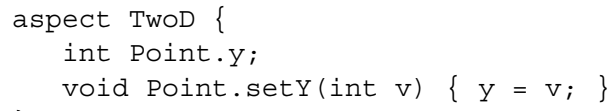

The command that composes class Point ${ }_{0}$ and aspect TwoD and achieves a program equivalent to Point ${ }_{1}$ is:

ajc Point 0 .java TwoD.java

Second increment. Aspect counter introduces field counter to class Point and advises the execution of all set methods to increment this counter ${ }^{2}$ :

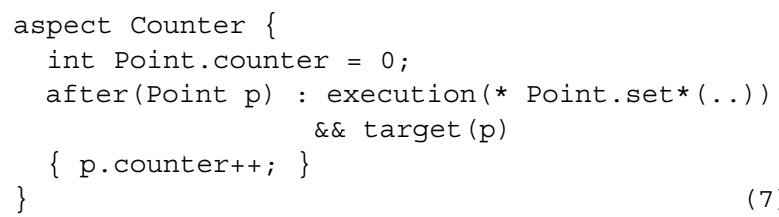

A program that is equivalent to Point 2 is produced by:

ajc Point,java TwoD.java Counter.java

Third increment. Aspect color adds a color field and a setColor method:

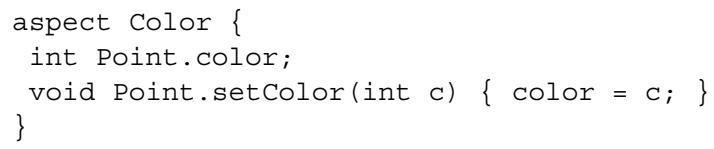

The composition of base with the three increments is:

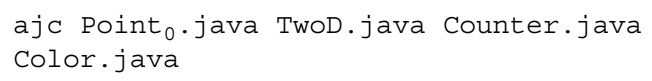

However, this time the result is not Point 3 , but instead:

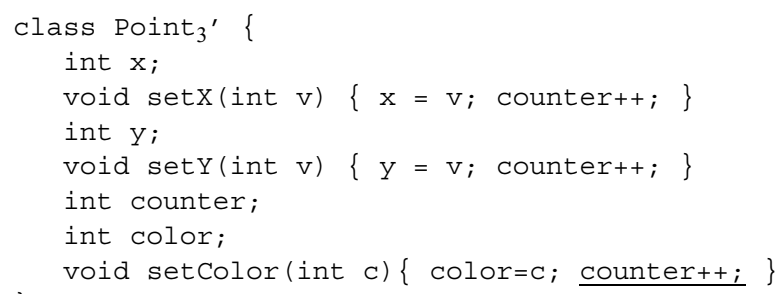

That is, the setcolor method of Point ${ }_{3}$ increments

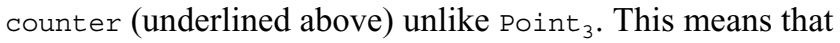
developers face the problem that building a program incrementally by hand may yield different results than using AspectJ. To produce Point ${ }_{3}$ using AspectJ, we should have used a more constrained version of counter that captures execution join points only of setX and setY methods:

2. In AspectJ there are other ways to define Counter. Shortly, we will present the rationale behind this implementation decision. 


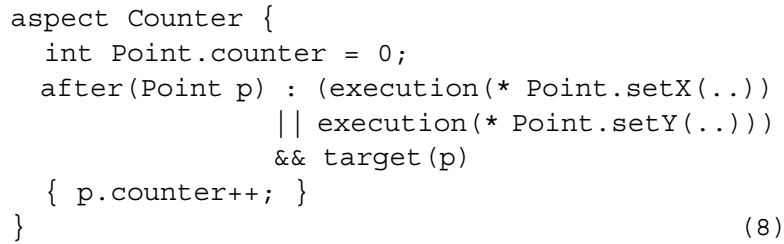

An obvious question is: why was counter not defined like (8) in the first place? Doing that certainly would solve this problem, but we must consider other properties of software modules that are also desirable for aspects. Among them is reusability, i.e., we want to treat aspects as components as in CBSE and reuse them as is. For example, suppose Counter is redefined as (8), but now we want to build program Point ${ }_{3}$ ' instead. We would have to revise counter back to (7) as the version in (8) cannot be used. The question is: why can we not reuse the same aspect for both cases? The problem is that aspect weaving does not distinguish among development stages of a program. We show how to solve this problem in the next section.

\section{An Algebraic Model of Aspects}

In this section, we develop an algebraic model that reveals another source of complexity in AspectJ composition. We then propose an alternative model of composition that retains the power of AspectJ, supports step-wise development, simplifies advice precedence, and facilitates reasoning using aspects. Our model has three operations that build upon the notions of introduction, advice, and weaving.

\subsection{Preliminaries}

Our model requires all method introductions to be explicit. Advice in AspectJ implicitly introduces a method. Recall the Logging aspect:

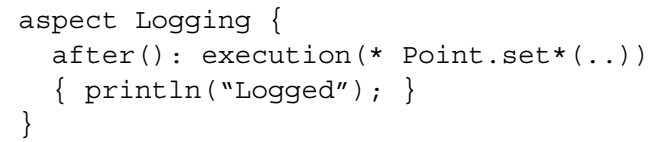

When woven into class Point ${ }_{0}$ in (6), aspect Logging can be regarded as the transformation that results in:

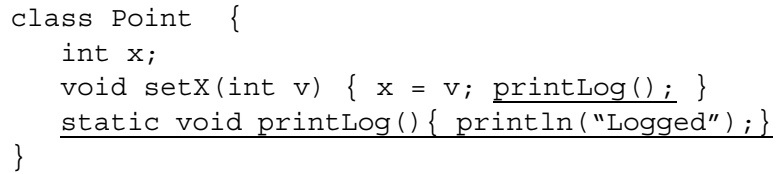

Method printLog is an explicit method that contains the advice body (the log message) and it is called at the end of the body of method setx (after the method execution).

To separate advice from introductions, pure advice is a named advice that replaces the advice body with a method call. To preserve AspectJ semantics, this call is not advisable, i.e., it has no join points. All join points of the original advice body reside in the method that is called. For example, we can conceptually rewrite the Logging aspect as:

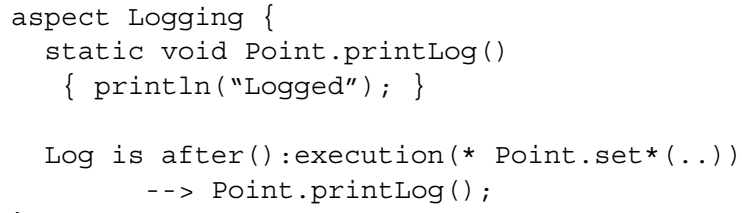

where Log is the name given to the pure advice, printLog is the method that contains the advice body, and the --> arrow indicates the method call. Other researchers have advocated a similar concept (that of making advice bodies into explicit methods), but have motivated the idea for different reasons [37]. Without loss of generality, we assume all advice is pure advice from this point on in this paper.

\subsection{Introduction Sum}

An introduction is a function that adds a data member or method to a program. Recall aspect TwoD and class Point whose composition was modeled algebraically as:

$$
\text { Point }_{1}=\operatorname{TwoD}\left(\text { Point }_{0}\right)
$$

where Point ${ }_{0}$ and Point ${ }_{1}$ are values, and TwoD is a function that maps class Point 0 to class Point ${ }_{1}$. Appealing to intuition, we can rewrite (10) as the sum of the introductions of TwoD with Point : $^{2}$

Point $_{1}=$ TwoD + Point $_{0}$

Operation + is called introduction sum. It is a binary operation that performs disjoint union on program fragments, which are sets of variables and methods. For example, aspect TwoD is the program fragment (set) containing y and sety, and class Point ${ }_{0}$ is the fragment (set) containing $\mathrm{x}$ and setx. We write introduction sum as:

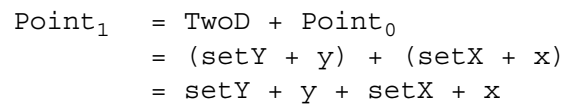

meaning Point ${ }_{1}=\{$ sety, $y, \operatorname{set} x, x\}$ where our notation above omits set brackets. As + is disjoint set union, introduction sum has the following properties:

Identity. 0 is the empty program (i.e., a program fragment that contains no members). If $\mathrm{x}$ is a program fragment:

$\mathrm{x}=\mathrm{x}+0=0+\mathrm{x}$

Commutativity. + is commutative because set union is commutative.

Associativity. + is associative as set union is associative.

+ differs from AspectJ introduction in that it does not allow member overriding. We believe overriding is rarely used and can be circumvented with a more structured design. ${ }^{3}$ 


\subsection{Weaving}

Pure advice is a function that maps an input program to a program where calls to advice methods have been inserted. We use function application to model the operation of weaving. Let a be pure advice and $\mathrm{P}$ be a program. The result of applying (weaving) a into $\mathrm{P}$ is program $\mathrm{P}^{\prime}$ :

$$
\mathrm{P}^{\prime}=\mathrm{a}(\mathrm{P})
$$

Weaving has the following properties:

Identity. id is the null pure advice - i.e., pure advice that captures no join points. Null pure advice is the identity transformation; its application does not affect a program. If $P$ is a program fragment, $P=i d(P)$. That is, $P$ does not change when woven with id.

Associativity. Weaving is right associative.

Distributivity. Weaving distributes over introduction sum. Let $P$ be a program, a be pure advice, and $P^{\prime}=a(P)$. Suppose $\mathrm{P}=\mathrm{X}+\mathrm{Y}+\mathrm{Z}$, where $\mathrm{X}, \mathrm{Y}$, and $\mathrm{Z}$ are arbitrary program fragments. We have:

$$
\begin{aligned}
P^{\prime} & =a(P) \\
& =a(X+Y+Z) \\
& =a(X)+a(Y)+a(Z)
\end{aligned}
$$

Advice applies to all join points in a program. Thus it is immaterial if the program fragment is viewed as a whole (P) or as the sum of its parts $(\mathrm{X}+\mathrm{Y}+\mathrm{Z})$. This distributivity property is central to AOP.

\subsection{Advice Sum}

Each piece of advice is a transformation (i.e., a function). The application of multiple pieces of advice is modeled by function composition, denoted by $\bullet$, which we call advice sum. • also models advice precedence. аз a1 means apply advice al first and then a3. Advice sum has the properties:

Identity. $i d$ is the null pure advice. If $a$ is pure advice:

$$
a=a \cdot i d=i d \bullet a
$$

Commutativity. The order in which advice is applied matters. $\bullet$ is not commutative. ${ }^{4}$

Associativity. - is associative because function composition is associative.

\subsection{Modeling Aspects as Pairs}

We model an aspect as a pair of two entries. The first entry, called the advice part, is the aspect's advice and the second

3. + also allows new classes and interfaces to be added to a program, which AspectJ does not support. This extra ability is useful when new functionality is added [32]. Aspects can encapsulate nested classes and nested interfaces, but not classes and interfaces that are unnested.

4. Two pieces of advice commute if they have no join point in common. entry, called the introduction part, is the aspect's introductions. The pair for Logging ( 9 ) is:

Logging $=<$ Log, printLog $>$

where Log is the name of the pure advice and printLog is the name of the introduced method.

Counter is another example. A pure advice version of it is:

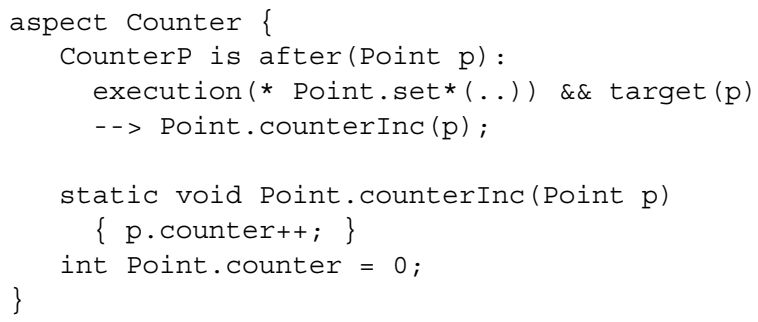

and its pair is:

Counter $=<$ Counter $\mathrm{P}$, counterInc + counter $>$

Note that the second entry of the pair sums the introductions counterInc (the method of the advice body) and counter (the variable).

Finally, a pure version of the Circular aspect (5) is:

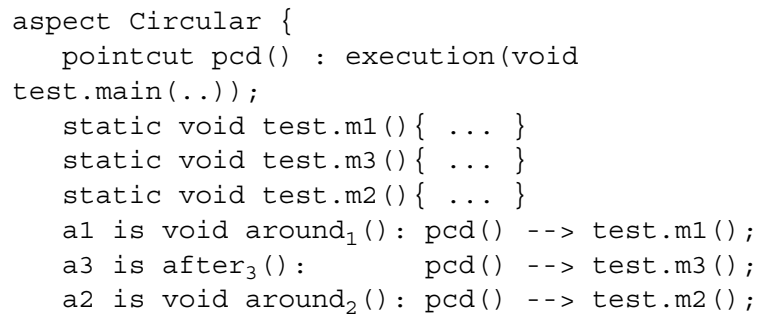

Suppose advice is woven in the textual order listed in an aspect. Circular would be modeled by the pair:

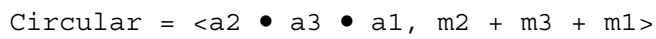

The expression a2 a $3 \bullet a 1$ represents compound advice, where $a_{1}$ is woven first and a2 last.

\subsection{Aspect Composition}

Let aspects $A 1$ and $A 2$ be modeled by the pairs $A 1=\left\langle a_{1}, i_{1}\right\rangle$ and $A 2=<a_{2}, i_{2}>$. We denote aspect composition in Aspect $J$ by operation $\diamond$. The AspectJ composition of A2 with A1 (with A1 being applied first) is:

$$
\begin{aligned}
\text { A2 } \diamond \mathrm{A} 1 & \left.=\left\langle\mathrm{a}_{2}, i_{2}\right\rangle\right\rangle\left\langle\mathrm{a}_{1}, i_{1}\right\rangle \\
& =\left\langle\mathrm{a}_{2} \bullet \mathrm{a}_{1}, i_{2}+i_{1}\right\rangle
\end{aligned}
$$

$\diamond$ is similar to vector addition because the coordinates of pairs are summed: + sums program fragments, • sums advice in weaving order.

As another example, program $\mathrm{P}$ can be modeled by the pair $\langle i d, p\rangle$, where $i d$ is null pure advice and $p$ is the introduc- 
tion sum of the members of P. Weaving aspect A1 into P and then weaving aspect $\mathrm{A} 2$ is:

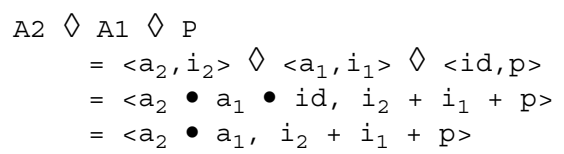

The code of a pair is the program that the pair represents. Let $\mathrm{v}$ be a pair. Its code, denoted [V], is computed by weaving its advice part with its introduction part:

$$
[V]=[<a, i>]=a(i)
$$

This follows from the fact that advice can advise any join point of a program ${ }^{5}$. Thus the program that is produced by weaving $A 1$ and then $A 2$ into $P$ is:

$$
[A 2 \diamond A 1 \diamond P]=a_{2} \bullet a_{1}\left(i_{2}+i_{1}+p\right)
$$

More generally, when aspects $A_{1} \ldots A_{n}$ are woven in this order into $\mathrm{P}$, the result is:

$$
\begin{aligned}
{\left[A_{n}\right.} & \left.\diamond A_{n-1} \diamond \ldots \diamond A_{1} \diamond P\right] \\
& =a_{n} \bullet a_{n-1} \bullet \ldots a_{1}\left(i_{n}+i_{n-1}+\ldots+i_{1}+p\right)
\end{aligned}
$$

That is, the result of weaving a sequence of aspects into a program equals the weaving of advice in weaving order into the program that is the introduction sum of the program's members and aspect introductions. (14) represents the "shape" of any program produced by AspectJ. We call this the pair model of composition.

(14) identifies the source of the problems noted earlier in Section 3 about incremental program development using AspectJ. It can be seen in the expansion of (13):

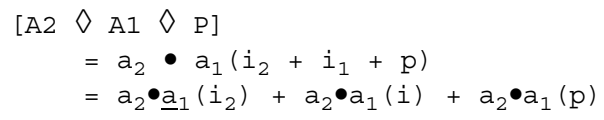

The offending term is underlined. It means that to apply aspect A2, the programmer is required to know how an advice from a previous development step $\left(a_{1}\right)$ affects an introduction added in the current step $\left(i_{2}\right)$. More generally, the weavings that cause problems in incremental development are underlined below:

$$
\ldots \bullet a_{\mathrm{k}+2} \bullet a_{\mathrm{k}+1} \bullet a_{\mathrm{k}} \bullet \underline{a}_{\mathrm{k}-1} \bullet \ldots \bullet \underline{a}_{2} \bullet \underline{a}_{1}\left(i_{\mathrm{k}}\right)+\ldots
$$

In other words, a programmer needs to know how previously applied pieces of pure advice $a_{j}$ affect later introductions $i_{k}$ where $j<k$. These are the terms that make stepwise development difficult. The problem is aggravated when a large number of aspects are composed and the development involves multiple steps.

5. Readers familiar with advice that advises itself will recognize that the pure advice part advises its introduction part. This is modeled by (12) .

\subsection{The Functional Model}

An alternative way to compose aspects is to equate aspect composition with function composition. Consider aspect $\mathrm{A}=<\mathrm{a}, \mathrm{i}>$. We can model $\mathrm{A}$ as the function:

$$
A(x)=a(i+x)
$$

That is, A adds its introductions ( $i$ ) to its program fragment input $(\mathrm{x})$ before weaving its advice (a). So applying aspect $\mathrm{A} 1$ to program $\mathrm{P}$ and then applying aspect $\mathrm{A} 2$ is:

$$
\begin{aligned}
\mathrm{A} 2(\mathrm{~A} 1(\mathrm{P})) & =\mathrm{a}_{2}\left(i_{2}+\mathrm{a}_{1}\left(i_{1}+\mathrm{p}\right)\right) \\
& =\mathrm{a}_{2}\left(i_{2}\right)+\mathrm{a}_{2} \bullet \mathrm{a}_{1}\left(i_{1}\right)+\mathrm{a}_{2} \bullet \mathrm{a}_{1}(\mathrm{p})
\end{aligned}
$$

Note that the offending pure advice $a_{1}$ disappears from the left summand $\left(a_{2}\left(i_{2}\right)\right)$. This generalizes to the composition of any number of aspects: the weavings that make step-wise development difficult are never generated. We call this the functional model. ${ }^{6}$ In effect, the pair model expresses unbounded quantification (i.e., the scope of advice extends over the entire program) [20], whereas the functional model expresses bounded quantification (i.e., the scope of advice extends over a stage in a program's development). An obvious question arises: which model is more expressive?

The functional model can express programs that the pair model cannot express, for example program (15). This expression cannot be produced by the pair model because all pieces of advice are woven into all introductions yielding program (13). Again, this is a consequence of the unbounded quantification of the pair model.

Unbounded quantification is a special case of bounded quantification. Think of quantifiers in first-order logic: the scope of a quantifier extends from its position in a logical formula to the right. Unbounded quantification means that the formula starts with the quantifier. In the functional model, a weaving expression is interpreted the same way: the influence of advice extends to the right and not to the left. Let us illustrate this with program (13) which requires unbounded quantification. To construct this program in the functional model, we decompose aspect A1 into a pair of aspects, one containing introduction $i_{1}$ and the other with advice $a_{1}$ :

$$
\begin{aligned}
& \mathrm{A} 1_{\text {intro }}(\mathrm{x})=i_{1}+\mathrm{x} \\
& \mathrm{A} 1_{\text {advice }}(\mathrm{x})=\mathrm{a}_{1}(\mathrm{x})
\end{aligned}
$$

Similarly for aspect A2:

$$
\begin{aligned}
& \mathrm{A} 2_{\text {intro }}(\mathrm{x})=i_{2}+\mathrm{x} \\
& \mathrm{A} 2_{\text {advice }}(\mathrm{x})=\mathrm{a}_{2}(\mathrm{x})
\end{aligned}
$$

To build (13), we weave the aspects with introductions to $P$ first and then the aspects with advice to get:

6. The functional model was inspired by AHEAD, which implements the laws of this paper; AHEAD has been successfully used to synthesize large systems [11]. 
$\mathrm{A} 2_{\text {advice }}\left(\mathrm{A} 1_{\text {advice }}\left(\mathrm{A} 2_{\text {intro }}\left(\mathrm{A} 1_{\text {intro }}(\mathrm{P})\right)\right)\right)$

$=a_{2} \bullet a_{1}\left(i_{2}+i_{1}+p\right)$

This ability to model unbounded quantification in the functional model is general, and is not specific to this particular example.

In summary, the functional model can express all programs that the pair model can express, and more once aspects are expressed in terms of bounded quantification. There are many useful programs that need bounded quantification. Recall the Point example of Section 3. Let us add a third dimension to Point, which is defined by aspect Threed that introduces a $z$ variable and setz method. Assuming that Counter advises all set methods as in (7) using bounded quantification, we can build at least four programs:

\footnotetext{
(a) Color (Threed (TwoD (Counter (Pointo $)$ )) )

(b) Color (ThreeD (Counter (TwoD (Point 0$)$ )))

(c) Color (Counter (Threed (TwoD (Point 0$)$ )))

(d) Counter (Color (Threed (TwoD (Point 0$)$ )) )
}

(a) is a program that counts the executions of setx. (b) counts the executions of setX and setY. (c) counts the executions of setX, setY, and setz. (d) counts the execution of all set methods. Each of these programs is synthesized by reusing and composing aspects as is. Using AspectJ, weaving aspects Counter, Color, ThreeD, and TwoD in an arbitrary order into P will always produce program (d). To build all four programs using AspectJ would require four different versions of counter.

To summarize, problems in step-wise development arise using AspectJ when pointcuts are not bounded to a set of classes, methods, and variables at a specific stage of program development. Common examples are pointcuts that capture the set of all calls to one or more methods, and wildcard patterns. Subsequent introductions that are captured by these pointcuts give rise to the problems discussed here. The functional model avoids these problems.

\section{Perspective}

We showed in Section 2.3 that precedence in AspectJ makes reasoning about and composing programs unnecessarily difficult. We showed in Section 4.7 that when aspects are reused as is, many programs cannot be built. We will show how to remove these limitations in Section 5.2 without sacrificing the power of AspectJ. Further, our work places aspects closer to key results in automated software design, which we now discuss.

\subsection{Automated Software Design}

The history of automated software design is replete with results on transformations and their connection to program structure. Tool-enabled program refactorings are program transformations [43]. Layers in layered software designs are transformations [10]. When viewed as increments in program functionality, features in software product-lines are transformations [11]. Model transformations play a key role in Model Driven Architectures; they are mappings between models (which are non-code representations of programs) [12]. Arguably the most significant result in automated software design is relational query processing [40]: a query evaluation program is defined by a composition of relational algebra operations. These operations are transformations of query evaluation programs.

Aspects define very useful transformations, and their strength is that programmers do not need understand transformation technologies to use them. As mentioned earlier, transformations are just one of a number of ways in which aspects can be implemented. The advantage of viewing aspects as transformations is that it exposes how aspects modify a program's structure. Doing so places aspects in context with results in software architectures and automated software development that also define and modify program structure.

\subsection{Impact on Existing Tools}

Our work eliminates several problems in AspectJ. First, the precedence rules for ordering pieces of advice within an aspect (Section 2.3) can be eliminated. We propose a simpler rule: apply advice in the order in which it is listed in an aspect file. This rule will simplify the ordering algorithms currently utilized by aspect compilers and will help AspectJ programmers by reducing the effort to determine a composition order.

Second, the rules that AspectJ uses to assign precedence to aspect files can also be eliminated. We propose that a precedence be declared for all aspect files to define their composition order. Alternatively, the compiler could raise an error when users fail to specify an order where ordering matters. Again, this change simplifies advice ordering algorithms for multiple aspect files and also helps programmers as now aspect compiler output will be predictable.

AOP researchers have raised the issue that it should be unnecessary to specify a composition order when aspects are provably commutative. We agree. In cases where pointcuts have disjoint sets of join points their corresponding advice is commutative. Existing tool support can help identify these situations [8]. However, it is still necessary to specify when these pieces of advice are to be applied. This may require an enhancement of existing tools.

\subsection{Related Work}

Since the Sixties, the paradigm of layered software has been applied to harness the complexity of large software systems (initially, they were operating systems [18]). A key property of layered software is that a layer has knowledge of lower layers but not of higher layers. (This is bounded 
quantification). We apply this principle to aspect orientation: the weaving of an advice affects the existing program, but not any parts that are added later.

Compositional models of aspects have a long history. GenVoca, AHEAD, and HyperJ are examples [10][11][20][42]. Relating compositional models to algebras is discussed in [11]. An early version of the ideas in this paper were presented at an AOSD 2005 workshop [33].

The relationship between program transformations and aspects is not new. Lämmel studied the implementation of aspects as programs transformations [30]. Kniesel et al. developed JMangler, a backend tool to support AOP that relies in transformations at the bytecode level [27]. The work of Krishnamurthi et al. on modular verification of aspect advice has an underlying assumption that aspects can be regarded as transformations [28]. We extend these ideas by showing how a transformation view leads to an algebraic model of aspect composition.

McEachen and Alexander consider the problems caused by weaving bytecode that already contains woven aspects [35]. A foreign aspect is an aspect that has been woven; the woven bytecode is later imported by a third party that has no access to the aspect's source code. A foreign aspect "comes alive" and can potentially affect subsequently added base or aspect code. Foreign aspects are problematic as they can: a) not capture all intended join points, b) capture unintended join points, and c) inadvertently interact with other aspects. The authors advocate guidelines to design the scope of pointcuts, use of abstract pointcuts to control the set of join points advisable by foreign aspects, and promote adequate pointcut documentation. Our work provides a foundation to understand the problems caused by foreign aspects and a solution to eliminate them. The rules of the functional model can be enforced by a compiler, whereas adhering to the guidelines of McEachen and Alexander is the responsibility of programmers.

Modular reasoning with AOP is controversial [15][16][17]. Kiczales and Mezini claim that in the presence of aspects "the complete interface of a module can only be determined once the complete configuration of modules in the system is known" [26]. In other words, aspects entail global reasoning that they define as "having to examine all the modules in the system or subsystems". The pair model of AspectJ mathematically corroborates their claim and shows the negative implications it has for incremental development. The functional model of composition reduces the need of global reasoning without restricting the power of AspectJ.

Rinard et al. propose a framework to classify aspects based on their interactions with other aspects and base code [38]. They present a tool that alerts users of cases where modular reasoning (a user-defined property) could be compromised so that users can take corrective action when necessary. Open modules proposes a module system whereby an interface describes the pointcuts and join points that are advisable by the pieces of advice of other modules thus promoting modular reasoning about aspects [1].

Complementary to our approach, there is work that aims to improve aspect reuse by making significant language changes. Gybels and Brichau propose a logic-based crosscut language to better decouple aspects from programs [22]. Rho and Kniesel propose aspect uniform genericity, application of logic metavariables in language constructs, as a way to promote reuse and to significantly expand the generic capabilities of AspectJ [39]. Incidentally, they too take a transformation view of aspects.

Classpects unify aspects and classes [37]; they are classes enhanced with bindings. A binding associates an advice type (before, after, around) and a pointcut with a call to a list of methods. These methods replace the advice body, similar to what we did when we transformed advice into pure advice.

A full implementation of the functional model requires advances in compilers. One issue is separate compilation [13][2], which is gaining attention in the programming languages community. Our need for separate compilation comes from the commutativity of operation + , which permits the introduction of a method before introducing other members on which the former may depend. This problem exists now in AspectJ because aspect files cannot be compiled separately from their base program. Also, a type theory is needed to tell us whether expressions are valid [25].

\section{Conclusions}

Aspect-oriented programming should be in the repertoire of tools and techniques used by software developers. But the current model and flagship tool of AOP, AspectJ, has limitations: aspect reuse is limited, woven programs can have hard to predict behavior, modular reasoning using aspects is difficult, and step-wise development of programs is errorprone. We explored these limitations and found that a significant source of complexity in AspectJ is its model of aspect composition.

To address these problems, we recognized that there are many ways in which aspects could be implemented. We selected a way - using program transformations - that revealed how aspects alter a program's structure. This allowed us to raise aspects from code artifacts to mathematical entities (functions that transform programs) and enabled us to develop an algebra to model aspect composition. Our algebra exposes a source of the current problems, it also reveals a solution. By equating aspect composition 
with function composition, the problems were eliminated and the power of AspectJ was preserved.

We are now investigating how to include other AspectJ capabilities such as declare parents, abstract aspects, abstract pointcuts, and aspect inheritance into our model. Our goal is to build languages and tools based on our model and to evaluate their potential in an experimental setting using the Aspect Bench Compiler (abc) [9][5]. We believe that our work lays an algebraic foundation on which to build and understand AOP tools.

Acknowledgements. We thank Gary Leavens, Paul Kelly, Oege de Moor, Axel Rauschmayer, Jim Cordy, Sven Apel, and Dewayne Perry for their comments on earlier drafts.

This research is sponsored in part by NSF's Science of Design Project \#CCF-0438786.

\section{References}

[1] J. Aldrich. Open Modules: Modular Reasoning about Advice. ECOOP 2005

[2] D. Ancona, G. Lagorio, and E. Zucca, "True Separate Compilation of Java Classes", PPDP 2002.

[3] AOSD Europe Network of Excellence Workshop. ECOOP 2005.

[4] AspectJ Developers mailing list. dev.eclipse.org/mhonarc/lists/aspectj-dev/maillist.html. Thread on execution order. October 5, 2005.

[5] P. Avgustinov, et al., "abc: An Extensible AspectJ Compiler", AOSD 2005, Chicago, USA

[6] P. Avgustinov, et al. "Optimizing AspectJ", PLDI 2005.

[7] AspectJ, version 1.2.1, eclipse.org/aspectj/.

[8] AspectJ Manual, www.eclipse.org/aspectj/doc/ progguide/language.html.

[9] Aspect Bench Compiler. www . aspectbench.org

[10] D. Batory and S. O'Malley. "The Design and Implementation of Hierarchical Software Systems with Reusable Components", ACM TOSEM, October 1992.

[11] D. Batory, J.N. Sarvela, A. Rauschmayer, "Scaling Step-Wise Refinement", IEEE TSE, June 2004.

[12] J. Bezivin, "From Object Composition to Model Transformation with the MDA", TOOLS'USA, August 2001.

[13] L. Cardelli, "Program Fragments, Linking, and Modularization", POPL 97.

[14] S. Chiba, "Program Transformation with Reflective and Aspect-Oriented Programming", in [31].

[15] C. Clifton and G. Leavens, "Observers and Assistants: A Proposal for Modular Aspect-Oriented Reasoning”, FOAL 2002.

[16] C. Clifton, G.T. Leavens. "Obliviousness, Modular Reasoning, and the Behavioral Subtyping Analogy". SPLAT 2003.

[17] C. Clifton, "A Design Discipline and Language Features for Modular Reasoning in Aspect-Oriented Programs", Ph.D. Dept. Computer Science, Iowa State, 2005.

[18] E.W. Dijkstra. "The Structure of the 'THE'-Multiprogramming System”, CACM, May 1968
[19] R. Dounce, D. Le Botlan. "Towards a Taxonomy of AOP Semantics". AOSD-Europe. Technical Report, July 2005.

[20] R.E. Filman, T. Elrad, S. Clarke, M. Aksit. Aspect-Oriented Software Development. Addison-Wesley, 2004.

[21] J. Gray et al. "A Technique for Constructing Aspect Weavers Using a Program Transformation Engine”. AOSD 2004.

[22] K. Gybels and J. Brichau, "Arranging Language Features for More Robust Pattern-based crosscuts", AOSD 2003.

[23] K Gybels and K. Ostermann, Discussions at SPLAT 2005.

[24] E. Hilsdale and J. Hugunin. "Advice weaving in AspectJ". AOSD 2004

[25] D. Hutchins, "Making Inheritance Scale: Towards a Theory of Deep Mixin Composition”, Univ. of Edinburgh, 2005.

[26] G. Kiczales, M. Mezini. “Aspect-Oriented Programming and Modular Reasoning”. ICSE 2005.

[27] G. Kniesel, et al. "JMangler - A Framework for Load-Time Transformation of Java Class Files". SCAM 2001.

[28] S. Krishnamurthi, K. Fisler, M. Greenberg. "Verifying Aspect Advice Modularity". FSE 2004.

[29] R. Laddad. AspectJ in Action. Practical Aspect-Oriented Programming. Manning, 2003.

[30] R. Lämmel, "Declarative Aspect-Oriented Programming", PEPM 1999.

[31] R. Lämmel, J. Saraiva, and J. Visser (Eds), Generative and Transformational Techniques in Software Engineering, 2005.

[32] R.E. Lopez-Herrejon, et al. "Evaluating Support for Features in Advanced Modularization Techniques". ECOOP 2005.

[33] R.E. Lopez-Herrejon and D. Batory. "Improving Incremental Development in AspectJ by Bounding Quantification", SPLAT Workshop, March 2005.

[34] H. Masuhara, G. Kiczales, "Modeling Crosscutting AspectOriented Mechanisms". ECOOP 2003.

[35] M. McEachen, R.T. Alexander. "Distributing Classes with Woven Concerns - An Exploration of Potential Fault Scenarios". AOSD 2005

[36] Partsch, H., Steinbrüggen, R.: Program Transformation Systems. ACM Computing Surveys, September (1983).

[37] H. Rajan, K.J. Sullivan, "Classpects: Unifying Aspect- and Object-Oriented Programming", ICSE 2005.

[38] M. Rinard, A. Salcianu, S. Bugrara. "A Classification System and Analysis for Aspect-Oriented Programs", FSE 2004.

[39] T. Rho, G. Kniesel. "LogicAJ - A Uniformly Generic Aspect Language." Submitted.

[40] P. Selinger, et al, "Access Path Selection in a Relational Database System", ACM SIGMOD 1979, 23-34.

[41] Semantic Designs. www. semdesigns. com/

[42] P. Tarr, H. Ossher, et al., "N Degrees of Separation: MultiDimensional Separation of Concerns", ICSE 1999.

[43] L. Tokuda and D. Batory. "Evolving Object-Oriented Designs with Refactorings" J. Automated Soft. Engr. 8, 2001.

[44] C. Szyperski, Component Software: Beyond Object-Oriented Programming, Addison-Wesley, 2002.

[45] M. Wand, et al., "A Semantics for Advice and Dynamic Join Points in Aspect Oriented Programming”, TOPLAS 2004.

[46] N. Wirth, "Program Development by Stepwise Refinement", CACM 14 \#4, 221-227, 1971. 